Original Paper http://ajol.info/index.php/ijbcs http://indexmedicus.afro.who.int

\title{
Performances agronomiques des arbres-mères d'anacardiers (Anacardium occidentale L.) sélectionnés dans les plantations paysannes au Bénin
}

\author{
Kouami N'DJOLOSSE ${ }^{1,2^{*}}$, Hubert ADOUKONOU-SAGBADJA ${ }^{2}$, Raphiou MALIKI $^{1}$, \\ Siaka KODJO $^{1}$, Antoine BADOU ${ }^{1}$ et René Nestor AHOYO ADJOVI ${ }^{3}$ \\ ${ }^{1}$ Centre de Recherches Agricoles Centre (CRA-Centre), Institut National des recherches Agricoles du \\ Bénin (INRAB), BP 112 Savè, Bénin. \\ ${ }^{2}$ Laboratoire des Ressources Génétiques et Amélioration Moléculaire des Espèces (LaReGAME), Faculté des \\ Sciences et Techniques (FAST), Université d'Abomey-Calavi (UAC), BP 1947, Abomey-Calavi, Benin. \\ ${ }^{3}$ Direction scientifique de l'Institut National des recherches Agricoles du Bénin (INRAB), 01 B P 884 Cotonou, \\ Bénin. \\ *Auteur correspondant ; E-mail : kndjolosse@yahoo.fr ; Tél. (+229) 96838341
}

\section{REMERCIEMENTS}

Les auteurs adressent leurs sincères remerciements à la Coopération Internationale Allemande (GIZ) pour avoir financé cette étude à travers son fonds de contrepartie (Matching Fund).

\section{RESUME}

L'une des contraintes majeures au développement de la culture de l'anacardier au Bénin est la non disponibilité de matériel végétal de plantation performant. L'objectif de cette étude était de sélectionner des arbres-mères d'anacardiers phénotypiquement supérieurs dans les plantations paysannes qui fourniront des greffons pour la production des plants greffés recommandés actuellement pour l'installation des vergers. Ainsi, une prospection a été réalisée dans 23 communes des 4 régions productrices de l'anacarde et appartenant aux zones agro-écologiques III, IV et V. Les arbres-mères potentiels identifiés ont été évalués entre 2013 et 2015 et les arbres performants ont été sélectionnés suivant trois principaux critères agronomiques : productivité en noix, poids des noix et taux d'amandes. Afin d'apprécier les performances des différentes catégories d'arbres-mères d'anacardiers par rapport à chaque paramètre mesuré, les données collectées ont été soumises à des analyses de la variance et à des comparaisons de moyennes. Au total, 357 arbres-mères d'anacardiers ont été sélectionnés sur les 1.868 arbres identifiés. Les productivités moyennes en noix des arbres-mères d'anacardiers sélectionnés ont été respectivement de 27,36 kg, 35,47 kg et 42,14 kg pour les tranches d'âges 5-9 ans, 10-15 ans et plus de 15 ans. Toutefois, on note une similarité entre les zones agro-écologiques quant aux trois paramètres évalués. Les résultats de cette étude constituent le point de départ d'un programme d'amélioration variétale de l'anacardier au Bénin.

(C) 2020 International Formulae Group. All rights reserved.

Mots clés : Amélioration variétale, anacardier, matériel végétal de plantation, productivité, Bénin. 


\title{
Agronomic performance of selected cashew (Anacardium occidentale $\mathbf{L}$.) mother trees in Benin farmer's plantations
}

\begin{abstract}
One of the major constraints to the cashew growing development in Benin is the unavailability of efficient planting material. The objective of this study was to select phenotypically efficient cashew mother trees in farmer's plantations which will provide scions for the production of grafted seedling currently recommended for orchards installation. Thus, a prospecting survey was carried out in 23 districts, in the 4 major cashew producing regions and belonging to agro-ecological zones III, IV and V. The potentials cashew mother trees which were identified were evaluated between 2013 and 2015 and the efficient mother trees were selected according to three main agronomic criteria: nut productivity, nut weight and kernel rate. In order to assess the performance of the different cashew mother tree categories in relation to each measured parameter, collected data were subjected to analysis of variance (ANOVA) and comparisons of means. A total of 357 cashew mother trees were selected from 1,868 identified potentials mother trees. The average nut productivity of the selected cashew mother trees were $27.36 \mathrm{~kg}, 35.47 \mathrm{~kg}$ and $42.14 \mathrm{~kg}$, respectively, for the age groups 5-9 years, 10-15 years and over 15 years. However, there is a similarity between agro-ecological zones with regard to the three evaluated parameters. The results of this study are the starting point for a cashew breeding program in Benin.
\end{abstract}

(C) 2020 International Formulae Group. All rights reserved.

Keywords: Breeding, cashew, planting material, productivity, Benin.

\section{INTRODUCTION}

L'anacardier est de nos jours une importante culture d'exportation pour le continent africain qui totalise plus de 55\% de la production mondiale de noix cajou. Les noix d'anacarde constituent un produit d'exportation de plus en plus demandé sur les marchés mondiaux et offrent un potentiel considérable pour le développement industriel des pays producteurs à travers la transformation locale. De même, le potentiel économique de l'anacarde offre des opportunités tout au long des chaînes de valeurs. La filière anacarde permet également d'apporter des réponses aux problématiques actuelles du changement climatique et de la migration (RONGEAD, 2015).

L'Afrique de l'Ouest est devenue la première zone de production de l'anacarde dans le monde depuis 2015 avec une production supérieure à 1350000 tonnes (RONGEAD, 2015). Au Bénin, la production en noix d'anacarde était de 110117 tonnes en 2017 (DSA/MAEP, 2017). Les superficies emblavées augmentent d'année en année. Elles ont été estimées en 2015 à 285.568 ha (Adégbola et Crinot, 2016). L'anacarde contribue à 3\% au Produit National Brut (PNB) et $7,4 \%$ au Produit Intérieur Brut Agricole (PIBA), ce qui la place en $2^{\text {ème }}$ position après la filière coton qui contribue à $25 \%$ au PIBA. Le Bénin dispose de vastes zones favorables à la culture de l'anacardier. Actuellement, l'anacarde est produit dans 5 des 8 zones agroécologiques du Bénin. Ces zones regroupent 8 des 12 départements du pays à savoir : Alibori, Atacora, Borgou, Collines, Couffo, Donga, Plateau et Zou (Adégbola et Crinot, 2016).

Malgré ces atouts, la productivité moyenne des anacardiers en noix cajou demeure très faible, 3 à $6 \mathrm{~kg} / \mathrm{arbre}$, par rapport à celle obtenue dans les pays grands producteurs du monde (10 à $15 \mathrm{~kg} / \mathrm{arbre})$ comme l'Inde, le Vietnam, le Brésil et la Tanzanie (Masawe, 2010; Tandjiékpon, 2010). Cette faible productivité des arbres affaiblit considérablement les productions et revenus des planteurs, des transformateurs, des commerçants et de l'Etat. Cette situation de faible productivité des anacardiers est due à plusieurs facteurs parmi lesquels on peut citer l'insuffisance ou l'inexistence de matériel végétal de plantation amélioré (MAEP, 2011).

C'est pour pallier cette insuffisance que des travaux de recherche sur l'amélioration variétale de l'anacardier ont démarré au Bénin 
en 2012. Cette étude qui est le point de départ de ces travaux présente les performances agronomiques des arbres-mères d'anacardiers identifiés et évalués. L'objectif visé était de sélectionner des arbres-mères d'anacardiers performants dans les plantations paysannes du Bénin. Ces arbres-mères d'anacardiers performants fourniront des greffons pour la production des plants greffés recommandés actuellement pour l'installation des vergers.

\section{MATERIEL ET METHODES \\ Milieu d'études}

La présente étude a été conduite dans les quatre principales régions favorables à la production de l'anacarde au Bénin. Il s'agit de : Atacora-Donga au Nord-Ouest, Borgou au Nord-Est, Zou-Collines et Plateau au Centre. Au total, 23 communes ont été couvertes. Cette zone est comprise entre les latitudes $7^{\circ} \mathrm{N}$ et $11^{\circ} \mathrm{N}$. La zone d'étude regroupe 3 des 8 zones agro-écologiques (ZAE) du Bénin : ZAE III, ZAE IV et ZAE V.

On y distingue trois (3) types de climats. La zone agro-écologique III regroupant le département du Borgou et deux communes de l'Atacora (Kouandé et Péhunco) a un climat de type soudanien avec une seule saison pluivieuse totalisant en moyenne entre 900 et $1300 \mathrm{~mm}$ de pluie par an. Le climat de la zone agro-écologique IV varie entre le soudanosahélien et le soudano-guinéen avec une pluiviométrie annuelle moyenne oscillant entre 1000 et $1300 \mathrm{~mm}$ par an. La zone agroécologique IV regroupe le département de l'Atacora sans les communes de Kouandé, Kérou et Péhunco puis le département de la Donga sans la communes de Bassila. La zone agro-écologique $\mathrm{V}$ regroupe le département des Collines plus les communes de Djidja, Kétou, Aplahoué, Bassila et Sud-Tchaourou. Elle a un climat de type soudano-guinéen à deux saisons pluvieuses au Sud et une saison pluvieuse au Nord avec une pluiviométrie annuelle moyenne variant entre 1000 et $1200 \mathrm{~mm}$ par an (Boko, 1992).

\section{Méthodologie}

Les méthodes utilisées dans cette étude sont celles décrites par Masawe (2010). Les méthodes utilisées pour chaque activité sont les suivantes :

Prospection dans les plantations paysannes d'anacardiers pour l'identification d'arbresmères

La prospection a été réalisée dans les quatre zones de grande production au Bénin : Atacora-Donga, Borgou, Zou-Collines et Plateau. Elle a couvert un total de 230 villages répartis dans 23 communes et 97 arrondissements (Tableau 1). Ainsi, des arbresmères d'anacardiers potentiels ont été identifiés dans les plantations paysannes suivant les critères définis par Masawe (2010). Les principaux critères de choix de ces arbresmères sont :

$\checkmark$ La productivité en noix qui est fonction de l'âge de l'arbre: un minimum de $20 \mathrm{~kg} /$ arbre par an est requis ;

$\checkmark$ La qualité des noix produites appréciée à travers le poids moyen d'une noix qui doit être supérieur à $6 \mathrm{~g}$ et le taux d'amandes supérieur à $25 \%$; et

$\checkmark$ l'état sanitaire: les arbres-mères doivent être indemnes de toutes attaques parasitaires.

Cette activité a été réalisée en période de floraison et de fructification (Masawe, 2010). Le Tableau 2 fournit des informations sur l'appréciation du rendement de l'anacardier en fonction de son âge.

\section{Évaluation des performances des arbres- mères d'anacardiers identifiés}

L'évaluation des performances productives des arbres-mères d'anacardiers potentiels identifiés a été réalisée sur 3 ans, de 2013 à 2015. Les paramètres clés de cette évaluation sont: la productivité en noix, la qualité des noix produites (poids moyen noix, poids moyen amandes, taux d'amandes) et l'état sanitaire. Les arbres présentant des symptômes de maladies et/ou des dégâts de ravageurs sont systématiquement rejetés.

Les noix produites par chacun des 1.868 arbres-mères potentiels identifiés ont été récoltées à chaque saison de production arbre par arbre et stockées dans des sacs de jute différents par les producteurs puis elles ont été pesées une fois par décade par les techniciens 
chargés de la collecte des données. La production totale est calculée par arbre à la fin de chaque saison pour avoir la productivité par arbre. Au milieu de la période de récolte de chaque saison de production, un échantillon de $1,5 \mathrm{~kg}$ de noix a été prélevé par arbre pour l'appréciation de leur qualité. Cette appréciation a été réalisée à la fin des récoltes sur un échantillon d'un kilogramme de noix apparemment saines. Le nombre de noix, les poids des noix et des amandes extraites après la coupe des noix ont été les données collectées.

\section{Analyses statistiques}

Le logiciel SPSS 16.0 a été utilisé pour les analyses statistiques. Afin d'apprécier les performances agronomiques des arbres-mères d'anacardiers identifiés et évalués en fonction de leurs tranches d'âges et des différentes zones agro-écologiques, des analyses de la variance ont été réalisées avec le modèle GLM (General Linear Model) et l'option des mesures répétées. Des comparaisons et des structurations des moyennes ont également été réalisées en utilisant la méthode de NEWMAN et KEULS.

Tableau 1 : Etendue de la prospection réalisée.

\begin{tabular}{|c|c|c|c|c|}
\hline Département & Commune & Arrondissement & $\begin{array}{l}\text { Nombre de } \\
\text { villages }\end{array}$ & Noms des villages \\
\hline \multirow{11}{*}{ Atacora } & \multirow{6}{*}{ Kouandé } & Birni & 1 & Birni-Pébirou \\
\hline & & Chabi Kouma & 2 & Chabi Kouma et Papatia \\
\hline & & Fô-Tancé & 1 & Fô-Tancé \\
\hline & & Guilmaro & 1 & Damouti \\
\hline & & Kouandé & 3 & $\begin{array}{l}\text { Becket-Bouramey, Maro et } \\
\text { Sékégourou (Mindou) }\end{array}$ \\
\hline & & Oroukayo & 3 & $\begin{array}{l}\text { Oroukayo, Niékéné-Bansou et } \\
\text { Ganikpérou }\end{array}$ \\
\hline & Sous-total 01 & 6 & 11 & \\
\hline & \multirow{3}{*}{ Péhunco } & Gnémasson & 1 & Sayakrou \\
\hline & & Péhunco & 4 & $\begin{array}{l}\text { Bouérou, Péhunco, Soassararou et } \\
\text { Sinaourarou }\end{array}$ \\
\hline & & Tobré & 6 & $\begin{array}{l}\text { Dakérérou, Guimbérérou, } \\
\text { Nimgoussourou, Gonri, } \\
\text { Ouassa-Maro et Tonri }\end{array}$ \\
\hline & Sous-total 02 & $\mathbf{3}$ & 11 & \\
\hline \multirow{11}{*}{ Borgou } & \multirow{4}{*}{ Bembèrèkè } & Bembèrèkè & 2 & Bembèrèkè Est et Kossou \\
\hline & & Bouanri & 1 & Bouanri \\
\hline & & Gamia & 2 & Bouay et Gamia-Ouest \\
\hline & & Ina & 2 & Ina 1 et Ina 3 \\
\hline & Sous-total 03 & 4 & 7 & \\
\hline & \multirow{4}{*}{ Kalalé } & Bouka & 2 & Gnèl-Wakibè et Gnèl-Yankan \\
\hline & & Dérassi & 2 & Alafiarou 2 et Dérassi \\
\hline & & Kalalé & 4 & $\begin{array}{l}\text { Bessassi, Djèga 2, Kalalé et } \\
\text { Kidaroukpérou }\end{array}$ \\
\hline & & Péonga & 1 & Péonga \\
\hline & Sous-total 04 & 4 & 9 & \\
\hline & N'Dali & N'Dali & 4 & $\begin{array}{l}\text { Sakarou, Sinisson, Suanin et Woba } \\
\text { Karou }\end{array}$ \\
\hline
\end{tabular}




\begin{tabular}{|c|c|c|c|c|}
\hline Département & Commune & Arrondissement & $\begin{array}{l}\text { Nombre de } \\
\text { villages }\end{array}$ & Noms des villages \\
\hline & & Sirarou & 2 & Maréborou et Sirarou \\
\hline & Sous-total 05 & 2 & 6 & \\
\hline & \multirow{4}{*}{ Nikki } & Gnonkourokali & 1 & Gnonkourokali \\
\hline & & Nikki & 4 & Danri, Gori Maro, Takou, Tontarou \\
\hline & & Ouénou & 1 & Ouénou \\
\hline & & Sérékalé & 2 & Kassakpéré et Sérékalé \\
\hline & Sous-total 06 & 4 & 8 & \\
\hline \multirow{17}{*}{ Borgou } & \multirow{3}{*}{ Parakou } & Parakou 1 & 2 & Tourou 1 et Tourou 2 \\
\hline & & Parakou 2 & 1 & Baka \\
\hline & & Parakou 3 & 1 & Dékparou \\
\hline & Sous-total 07 & $\mathbf{3}$ & 4 & \\
\hline & \multirow{4}{*}{ Pèrèrè } & Gninsy & 2 & Boro, Gninsy \\
\hline & & Guinagourou & 2 & Guinagourou et Nassy \\
\hline & & Kpébié & 1 & Kpébié \\
\hline & & Sontou & 2 & Alafiarou et Sontou \\
\hline & Sous-total 08 & 4 & 7 & \\
\hline & \multirow{2}{*}{ Sinendé } & Fô-Bouré & 2 & Fô-Bouré et Toumé \\
\hline & & Sikki & 2 & Sikki-Maro et Sikki-Gourou \\
\hline & Sous-total 09 & 2 & 4 & \\
\hline & \multirow{4}{*}{ Tchaourou } & Alafiarou & 2 & Agbassa, Alafiarou \\
\hline & & Goro & 1 & Goro \\
\hline & & Tchaourou & 2 & Koro et Papanè \\
\hline & & Tchatchou & 1 & Tchatchou \\
\hline & Sous-total 10 & 4 & 6 & \\
\hline \multirow{15}{*}{ Collines } & \multirow{6}{*}{ Bantè } & Agoua & 1 & N'Tchon \\
\hline & & Akpassi & 2 & Banon, Okoto \\
\hline & & Bantè & 4 & $\begin{array}{l}\text { Adjantè, Gbégamey, Illélakoun et } \\
\text { Basson }\end{array}$ \\
\hline & & Bobè & 1 & Bobè \\
\hline & & Gouka & 2 & Gouka et Montèwo \\
\hline & & Pira & 2 & Adjigo et Pira \\
\hline & Sous-total 11 & 6 & 12 & \\
\hline & & Gbaffo & 3 & $\begin{array}{l}\text { Gbaffo, Dovi-Somè et } \\
\text { Gnonkpingnon }\end{array}$ \\
\hline & & Kèrè & 3 & Gamba, Igoho et Odo-Otchèrè \\
\hline & Dassa-Zoumé & Kpingni & 1 & Togon \\
\hline & & Paouignan & 3 & $\begin{array}{l}\text { Adjanoudoho, Davissogo et } \\
\text { Paouignan }\end{array}$ \\
\hline & & Soclogbo & 3 & Akoba, Miniffi et Soclogbo \\
\hline & Sous-total 12 & 5 & 13 & \\
\hline & & Assanté & 1 & Gbanlin-Hansoé \\
\hline & Glazoue & Gomé & 1 & Gomé \\
\hline
\end{tabular}




\begin{tabular}{|c|c|c|c|c|}
\hline Département & Commune & Arrondissement & $\begin{array}{l}\text { Nombre de } \\
\text { villages }\end{array}$ & Noms des villages \\
\hline & & Kpakpaza & 1 & Kpakpaza \\
\hline & & Magoumi & 2 & Houala et Monso \\
\hline & & Ouèdèmè & 3 & Goto, Kpota et Ouèdèmè \\
\hline & & Thio & 6 & $\begin{array}{l}\text { Agouagon, Assromihoué, } \\
\text { Dokoundji, Kpassali et Riffo }\end{array}$ \\
\hline & Sous-total 13 & 6 & 14 & \\
\hline \multirow{18}{*}{ Collines } & \multirow{5}{*}{ Ouessè } & Challa-Ogoï & 4 & $\begin{array}{l}\text { Agboro-Kombon, Challa-Ogoï, } \\
\text { Idouya et Kokoro }\end{array}$ \\
\hline & & Gbanlin & 3 & Gbanlin, Gbeffadji et Tédohorindji \\
\hline & & Kèmon & 3 & Akpéro, Ekpa et Kèmon \\
\hline & & Ouessè & 3 & Gbémè, Ouessè, Zagba-Gaou \\
\hline & & Toui & 3 & Malété, Toui-PK et Toui-Gare \\
\hline & Sous-total 14 & 5 & 16 & \\
\hline & \multirow{5}{*}{ Savalou } & Gobada & 1 & Lama \\
\hline & & Kpataba & 2 & Koutago, Mondji \\
\hline & & Lahotan & 1 & Damè \\
\hline & & Savalou-Aga & 4 & $\begin{array}{l}\text { Kpakpassa, Lowo-Zoungo, } \\
\text { Honnoukon/Daga-Doho, Sohèdji }\end{array}$ \\
\hline & & Savalou-Attaké & 2 & Azokangoudo, Doïssa \\
\hline & Sous-total 15 & 5 & 10 & \\
\hline & \multirow{5}{*}{ Savè } & Kaboua & 2 & Gogoro, Okounfo \\
\hline & & Ofè & 2 & Dani, Gobé \\
\hline & & Okpara & 2 & Bessé-Ayédé, Oké-Owo \\
\hline & & Plateau & 1 & Fidégnon \\
\hline & & Sakin & 2 & Ogonla et Ouoghi \\
\hline & Sous-total 16 & 5 & 9 & \\
\hline \multirow{11}{*}{ Donga } & \multirow{3}{*}{ Bassila } & Bassila & 8 & $\begin{array}{l}\text { Adjégounlè, Adjiro, Baka-Baka, } \\
\text { Diépami, Biguina 1, Frignon, } \\
\text { Kikélé et Koïwali }\end{array}$ \\
\hline & & Manigri & 2 & Manigri-Oké et Manigri-Ikani \\
\hline & & Pénessoulou & 2 & Kodowari et Nagayilé \\
\hline & Sous-total 17 & 3 & 12 & \\
\hline & \multirow{3}{*}{ Copargo } & Anandana & 2 & Landa et Tchalougou \\
\hline & & Copargo & 4 & $\begin{array}{l}\text { Copargo, Kparakouwa, Toungouri } \\
\text { et Zongo }\end{array}$ \\
\hline & & Pabégou & 3 & $\begin{array}{l}\text { Bom-Bom, Nantayackou } 1 \text { et } \\
\text { Pabégou }\end{array}$ \\
\hline & Sous-total 18 & 3 & 9 & \\
\hline & \multirow{3}{*}{ Djougou } & Barei & 4 & $\begin{array}{l}\text { Bandétchori, Barei, Barei-Tanjar et } \\
\text { Gondessar }\end{array}$ \\
\hline & & Bariénou & 3 & $\begin{array}{l}\text { Affon-Gosso, Akèkèrou et } \\
\text { Toko-Toko }\end{array}$ \\
\hline & & Belléfougou & 1 & Belléfougou \\
\hline
\end{tabular}




\begin{tabular}{|c|c|c|c|c|}
\hline Département & Commune & Arrondissement & $\begin{array}{l}\text { Nombre de } \\
\text { villages }\end{array}$ & Noms des villages \\
\hline \multirow{12}{*}{ Donga } & & Djougou II & 4 & $\begin{array}{l}\text { Djakpingou, Kpamalangou, } \\
\text { Sérobouta et Sinassingou }\end{array}$ \\
\hline & & Kolokondé & 2 & Foumbéa, Kolokondé \\
\hline & & Onklou & 2 & Bakou, Daringa \\
\hline & & Pélébina & 3 & $\begin{array}{l}\text { Goumbakou, Faka-faka, Wassa } \\
\text { (Bata-Fata Fou) }\end{array}$ \\
\hline & Sous-total 19 & 7 & 19 & \\
\hline & \multirow{6}{*}{ Ouaké } & Badjoudé & 1 & Bohomdo \\
\hline & & Komdé & 3 & Adjede, Komdé et Wèkètè \\
\hline & & Ouaké & 4 & $\begin{array}{l}\text { Awanla, Sonatè, Sonaholou et } \\
\text { Tchaladè }\end{array}$ \\
\hline & & Sèmèrè I & 1 & Gnalo \\
\hline & & Sèmèrè II & 4 & Awotobi, Gbaou, Itchelli et Koubli \\
\hline & & Tchalinga & 2 & Madjatom et Tchalinga \\
\hline & Sous-total 20 & 6 & 15 & \\
\hline \multirow{4}{*}{ Plateau } & \multirow{3}{*}{ Kétou } & Adakplamè & 4 & $\begin{array}{l}\text { Adakplamè, Agozoumè, Ewè et } \\
\text { Sodji }\end{array}$ \\
\hline & & Idigny & 4 & $\begin{array}{l}\text { Atanagbalè, Araromi, Idjikodé et } \\
\text { Iladji }\end{array}$ \\
\hline & & Okpomèta & 2 & Issalè, Oké-Agbé \\
\hline & Sous-total 21 & $\mathbf{3}$ & $\mathbf{1 0}$ & \\
\hline \multirow{10}{*}{ Zou } & \multirow{4}{*}{ Djidja } & Agouna & 3 & Agouna, Dénou et Sankpiti \\
\hline & & Dan & 3 & Dan, Danoukpota et Tchakaladji \\
\hline & & Djidja & 2 & Madjavi I et Sègoun \\
\hline & & Monsourou & 2 & Amakpa et Kakatéou \\
\hline & Sous-total 22 & 4 & 10 & \\
\hline & \multirow{3}{*}{ Zangnanado } & Agonli-Houégbo & 1 & Houégbo-do \\
\hline & & Banamè & 5 & $\begin{array}{l}\text { Assiangbomè, Gbatèzoumè, } \\
\text { Gossoé, Houédanougon et Towégo }\end{array}$ \\
\hline & & Don-Tan & 2 & Don-Aliho , Don-Tohomè \\
\hline & Sous-total 23 & $\mathbf{3}$ & 8 & \\
\hline & TOTAL & 97 & 230 & \\
\hline
\end{tabular}

Tableau 2 : Base d'appréciation de la productivité en noix de l'anacardier en fonction de son âge.

\begin{tabular}{cccccc}
\hline \multirow{2}{*}{ Age (ans) } & \multicolumn{5}{c}{ Productivité annuelle en noix $(\mathbf{k g})$} \\
\cline { 2 - 6 } & $\mathbf{1 0}$ & $\mathbf{1 0 - 1 9}$ & $\mathbf{2 0 - 2 9}$ & $\mathbf{3 0 - 3 9}$ & $\geq \mathbf{4 0}$ \\
\hline $\mathbf{1 0}$ & Bon & Très bon & Meilleur & Meilleur* & Meilleur** \\
$\mathbf{1 0 - 1 5}$ & Mauvais & Bon & Très bon & Meilleur & Meilleur* \\
$>\mathbf{1 5}$ & Mauvais & Bon & Bon & Très bon & Meilleur \\
\hline
\end{tabular}

Source : Adapté de Masawe (2010) et Salifou \& Masawe (2018). 


\section{RESULTATS}

Caractéristiques agronomiques des arbresmères d'anacardiers identifiés

Les caractérististiques agronomiques des arbres-mères d'anacardiers potentiels identifiés sont résumées dans le Tableau 3. D'après ce tableau, la productivité moyenne des arbres-mères potentiels a augmenté avec l'âge des anacardiers. Par contre, la qualité des noix n'a pas sensiblement varié d'une tranche d'âge à l'autre. Cela a été perceptible à travers les valeurs quasi identiques des poids moyens des noix et des taux d'amandes.

Au terme de trois années d'évaluations, de 2013 à 2015, un total de 357 arbres-mères d'anacardiers performants ont été sélectionnés dans l'ensemble de la zone d'études sur la base des quatre principaux critères utilisés. Ces arbres-mères d'anacardiers performants sont distribués dans toutes les trois zones agroécologiques dans lesquelles le travail a été réalisé (Figure 1). On note une similarité entre les zones agro-écologiques par rapport aux caractéristiques agronomiques des arbresmères d'anacardiers performants sélectionnés.
Productivité en noix des arbres-mères d'anacardiers sélectionnés

Les résultats de l'analyse de la variance ont montré une différence significative entre la productivité en noix des arbres-mères d'anacardiers sélectionnés en fonction des tranches d'âges. Les productivités moyennes en noix des arbres-mères d'anacardiers sélectionnés ont été respectivement de $27,36 \mathrm{~kg}, \quad 35,47 \mathrm{~kg}$ et $42,14 \mathrm{~kg}$ pour les tranches d'âges 5-9 ans, 10-15 ans et plus de 15 ans $\quad(\mathrm{P}<0,000) \quad$ (Figure 2). On note cependant une similarité entre les zones agroécologiques en ce qui concerne la productivité des arbres-mères.

Qualité des noix produites par les arbresmères d'anacardiers sélectionnés

Les résultats de l'analyse de la variance ont montré une similarité entre les zones agroécologiques $(\mathrm{P}=0,128)$ et entre les tranches d'âges $(\mathrm{P}=0,345)$ quant à la qualité des noix produites par les arbres-mères d'anacardiers sélectionnés (Tableau 4). Les taux d'amandes moyens en fonction des zones agroécologiques ont varié de $28,10 \%$ à $29,33 \%$ et les poids unitaires moyens des noix ont varié de $6,35 \mathrm{~g}$ à $8,46 \mathrm{~g}$ dans les mêmes zones.

Tableau 3 : Caractéristiques agronomiques des arbres-mères d'anacardiers potentiels identifiés.

\begin{tabular}{ccrrrrr}
\hline $\begin{array}{c}\text { Tranches } \\
\text { d'âges des } \\
\text { arbres (ans) }\end{array}$ & $\begin{array}{c}\text { Statistiques } \\
\text { descriptives }\end{array}$ & $\begin{array}{c}\text { Ages des } \\
\text { arbres } \\
\text { (ans) }\end{array}$ & $\begin{array}{c}\text { Productivité } \\
\text { en noix (kg) }\end{array}$ & $\begin{array}{c}\text { Nombre } \\
\text { de noix } \\
\text { dans 1 kg }\end{array}$ & $\begin{array}{c}\text { Poids } \\
\text { unitaire } \\
\text { moyen des } \\
\text { noix (g) }\end{array}$ & $\begin{array}{c}\text { Taux } \\
\text { d'amandes } \\
(\%)\end{array}$ \\
\hline \multirow{2}{*}{$\mathbf{1 0}$} & Maximum & 9,0 & 57,00 & 316,7 & 10,10 & 35,64 \\
& Moyenne & 7,3 & 27,20 & 150,8 & 6,63 & 28,60 \\
& Minimum & 4,0 & 5,60 & 99,0 & 3,16 & 19,75 \\
\hline \multirow{2}{*}{$\mathbf{1 0 ~ - 1 5}$} & Maximum & 15,0 & 105,80 & 321,6 & 10,07 & 37,66 \\
& Moyenne & 12,6 & 33,42 & 151,2 & 6,61 & 28,49 \\
& Minimum & 10,0 & 9,60 & 99,3 & 3,11 & 17,60 \\
\hline & Maximum & 70,0 & 123,00 & 323,0 & 9,67 & 44,55 \\
& Moyenne & 21,7 & 38,26 & 156,9 & 6,37 & 28,67 \\
& Minimum & 16,0 & 8,70 & 103,4 & 3,10 & 14,71 \\
\hline
\end{tabular}




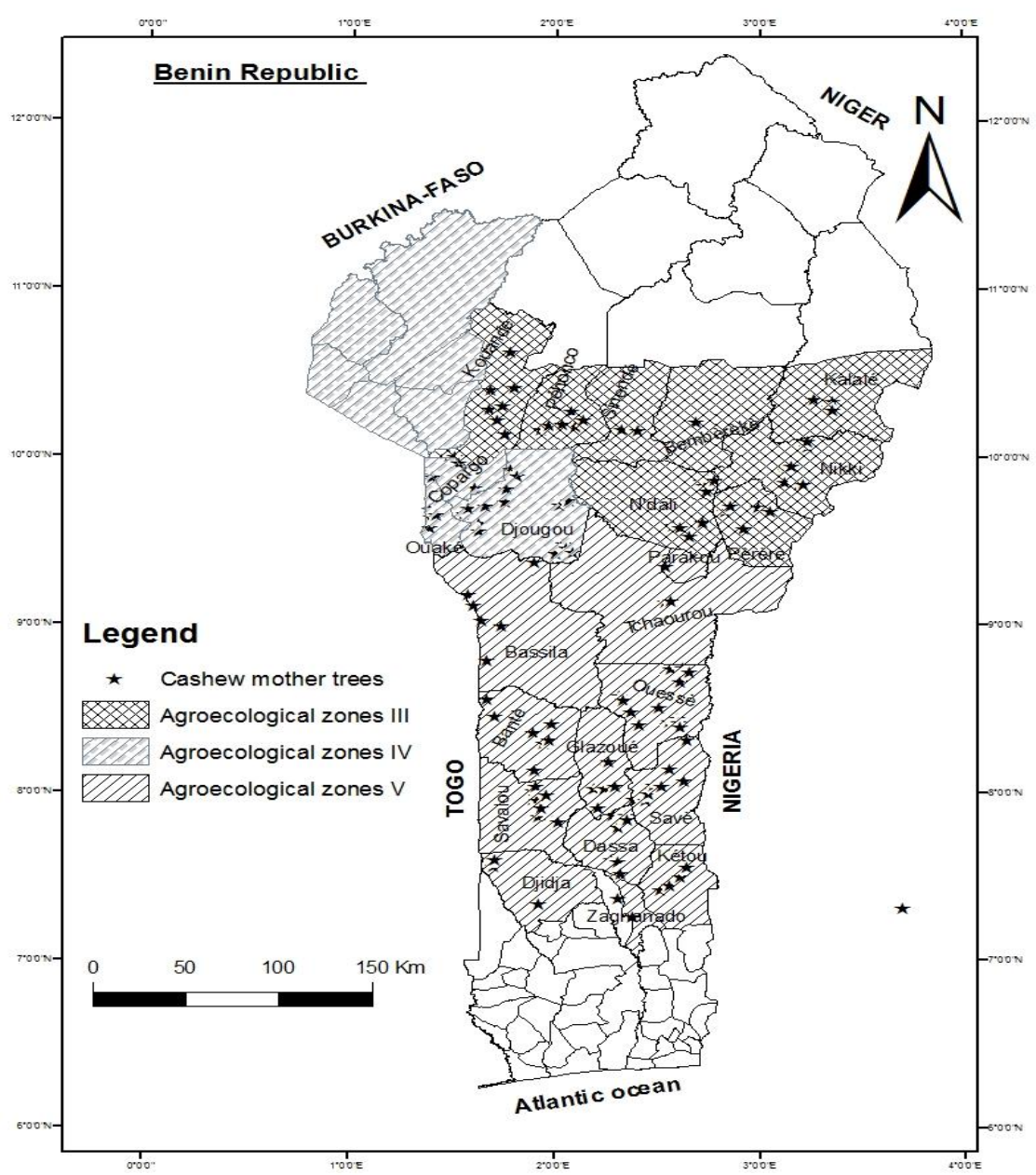

Figure 1 : Distribution des arbres-mères d'anacardiers sélectionnés dans la zone d'études.

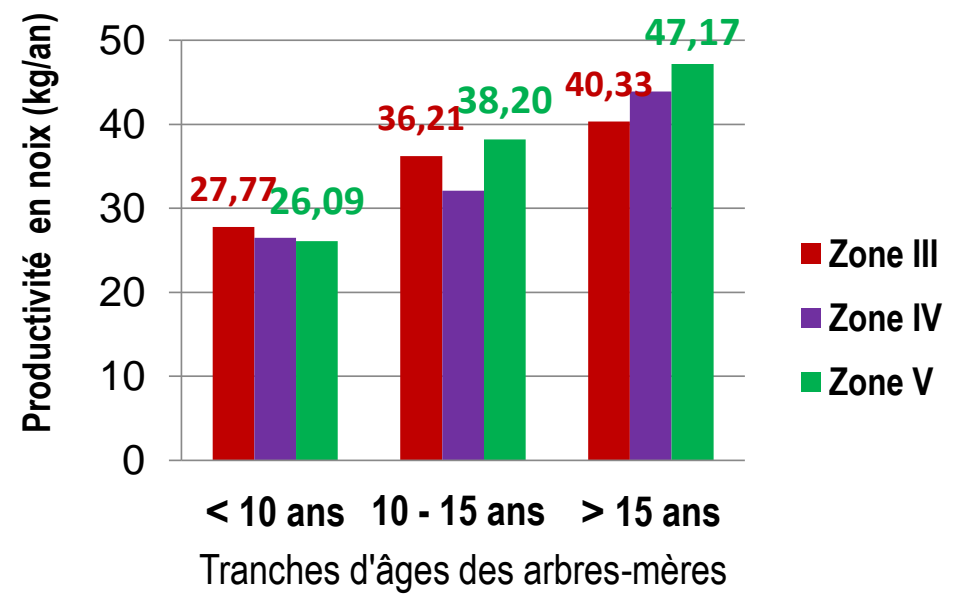

Figure 2: Productivités moyennes des arbres-mères d'anacardiers sélectionnés en fonction des tranches d'âges dans les trois zones agro-écologiques. 
Tableau 4 : Poids unitaire et taux d'amandes des noix produites par les arbres-mères d'anacardiers sélectionnés.

\begin{tabular}{lcccccc}
\hline Zones agro- & \multicolumn{2}{c}{ Taux d'amandes moyens (\%) } & \multicolumn{3}{c}{ Poids unitaire moyens des noix $(\mathrm{g})$} \\
\cline { 2 - 7 } écologiques & $<\mathbf{1 0}$ ans & $\mathbf{1 0} \mathbf{- 1 5}$ ans & $>\mathbf{1 5}$ ans & $<\mathbf{1 0}$ ans & $\mathbf{1 0} \mathbf{- 1 5}$ ans & $>\mathbf{1 5}$ ans \\
\hline Zone III & 28,49 & 28,10 & 28,90 & 7,10 & 7,18 & 6,91 \\
Zone IV & 28,69 & 29,33 & 28,98 & 8,03 & 7,28 & 8,46 \\
Zone V & 28,52 & 28,87 & 29,12 & 6,35 & 6,86 & 7,22 \\
\hline
\end{tabular}

\section{DISCUSSION}

Les productivités moyennes des arbresmères d'anacardiers potentiels identifiés ont varié de 27,20 à $38,26 \mathrm{~kg} / \mathrm{an}$ en fonction des tranches d'âges. Ces productivités moyennes obtenues sont toutes supérieures à productivité moyenne d'un anacardier ordinaire qui est de $15 \mathrm{~kg}$ de noix par an (Aliyu, 2004). Ceci confirme que ces arbres-mères identifiés sont de meilleurs anacardiers.

Les productivités des arbres-mères sélectionnés satisfont aux productivités minimales requises définies par Masawe (2010) et Salifou et Masawe (2018). Ainsi, les arbres-mères d'anacardiers sélectionnés ont une productivité moyenne atteignant au moins deux fois leurs âges (Salifou et Masawe, 2018). Les présents travaux ont permis de découvrir l'existence au Bénin des anacardiers très performants produisant plus de $120 \mathrm{~kg}$ de noix par an comme au Ghana et en Tanzanie (Yidana, 2003 ; Salifou et Masawe, 2018). De même, les qualités des noix produites par ces arbres-mères d'anacardiers sélectionnés traduites à travers les poids unitaires moyens des noix et les taux d'amandes moyens sont également conforment aux normes exigées pour les arbres-mères d'anacardiers (Salifou et Masawe, 2018).

$\mathrm{Au}$ total, seulement 357 arbres-mères d'anacardiers ont été sélectionnés sur les 1.868 arbres identifiés, soit un taux de $19,11 \%$. Ce faible taux d'arbres éligibles aux critères des arbres-mères d'anacardiers pourrait s'expliquer par les faibles productivités et les faibles poids unitaires des noix enregistrés sur beaucoup d'arbres identifiés. En effet, d'après Bello et al. (2017), les changements climatiques actuels affectent négativement les productions d'anacarde au Bénin. Selon
Amoussou et al. (2016), les précipitations ont connu une baisse significative variant de 2 à $13 \%$ dans le milieu de l'étude sur la période allant de 1981 à 2010 comparativement à la période allant de 1951 à 1980.

Les résultats des présents travaux montrent la possibilité de la mise en place d'un programme d'amélioration variétale de l'anacardier au Bénin sur la base des arbresmères d'anacardiers sélectionnés. Cela passera par leur caractérisation aux plans agromorphologique et génétique afin d'identifier les groupes phénotypiques et génétiques exploitables à des fins de création variétale.

\section{Conclusion}

Les résultats de cette étude ont montré l'existence d'arbres-mères d'anacardiers performants dans les plantations paysannes de toutes les trois zones agro-écologiques dans lesquelles le travail a été réalisé. Un total de 357 arbres-mères performants d'anacardiers ont été sélectionnés dans l'ensemble de la zone d'études pour exploitation dans un programme d'amélioration variétale de l'anacardier au Bénin. Ces arbres-mères d'anacardiers ont des productivités moyennes variant entre 26,09 et $47,17 \mathrm{~kg}$ de noix par an. Certains de ces arbresmères d'anacardiers produisent plus de $100 \mathrm{~kg}$ de noix par an. Les noix produites par ces arbres-mères ont des taux d'amandes moyens variant de $28,10 \%$ à $29,33 \%$ et des poids unitaires moyens variant entre $6,35 \mathrm{~g}$ et $8,46 \mathrm{~g}$. Ces arbres-mères d'anacardiers performants sélectionnés serviront à la fois à fournir des greffons pour la production de plants greffés destinés à l'installation de nouvelles plantations et à l'installation de germoplasmes et parcs à bois d'anacardiers nécessaires au programme d'amélioration variétale. 


\section{CONFLIT D'INTERETS}

Les auteurs déclarent qu'il n'y a aucun conflit d'intérêts pour la publication de cet article.

\section{CONTRIBUTIONS DES AUTEURS}

La conception du protocole de recherche a été réalisée par KN, SK et RM. Le suivi de la collecte des données a été assuré par $\mathrm{KN}, \mathrm{SK}, \mathrm{RM}, \mathrm{AB}$ et HAS. La rédaction de l'article a été réalisée par KN et $\mathrm{AB}$. Sa lecture a été assurée par RM, HAS et RNAA.

\section{REMERCIEMENTS}

Les auteurs témoignent leur gratitude aux autorités de l'INRAB, des ex-CARDER Atacora-Donga, Borgou-Alibori et ZouCollines pour les facilités administratives sans oublier les techniciens de ces CARDER qui ont énormément contribué à la collecte des données. Ils adressent également leurs sincères remerciements aux Reviewers pour leurs diverses contributions à l'amélioration de la qualité de cet article.

\section{REFERENCES}

Adégbola PY, Crinot G. 2016. Recensement des producteurs d'anacarde, des vergers d'anacardiers et des unités de transformation de cajou au Bénin. Rapport technique, Ministère de l'Agriculture, de l'Elevage et de la Pêche (MAEP), Programme Cadre d'Appui à la Diversification Agricole (ProCAD), Projet d'Appui à la Diversification Agricole (PADA), Janvier 2016, p. 59.

Amoussou E, Totin Vodounon SH, Clédjo FP, Allagbé YBS, Akognongbé JSA, Houndénou C, Mahè G, Camberlin P, Boko M, Pérard J. 2016. Evolution climatique du Bénin de 1950 à 2010 et son influence sur les eaux de surface. Présentation au XXIX ${ }^{\mathrm{è}}$ Colloque de l'Association Internationale de Climatologie: «Climat et pollution de l'air » du 06 au 09 juillet 2016 à Besançon (France), p.19. http://www.climato.be/aic/colloques/Lau sanneBesancon16/Pres/Amoussou.pdf
Aliyu OM. 2004. Characterization and compatibility studies in cashew (Anacardium occidentale L.). $\mathrm{PhD}$ Thesis, University of Ilorin, Ilorin, p. 266.

Bello DO, Ahoton EL, Saïdou A, Akponikpè IPB, Ezin VA, Balogoun I, Aho N. 2017. Climate change and cashew (Anacardium occidentale L.) productivity in Benin (West Africa): perceptions and endogenous measures of adaptation. Int. J. Biol. Chem. Sci., 11(3): 924-946. DOI: https://dx.doi.org/10.4314/ijbcs.v11i3.1

Boko M. 1992. Saisons et types de temps au Bénin : analyse objective et perceptions populaires. Espace Géographique, 21(4): 321-333. DOI : 10.3406/spgeo.1992.3106

Direction de la Statistique Agricole du Ministère de l'Agriculture, de l'Elevage et de la Pêche (DSA/MAEP). 2017. Rapport de l'enquête d'estimation du rendement de l'anacarde au Bénin. MAEP. ProCAD/PADA. Cotonou, p. 55.

Masawe PAL. 2010. Consultancy Report on Cashew Improvement Programme for Selected West African Countries (Benin, Burkina and Côte d'Ivoire), GIZ/iCA, Accra, p. 54.

Ministère de l'Agriculture, de l'Elevage et de la Pêche (MAEP). 2011. Plan Stratégique de Relance du Secteur Agricole (PSRSA), p. 116.

RONGEAD. 2015. Le bilan paradoxal de la campagne de noix de cajou 2015 en Afrique de l'Ouest. http://www.commodafrica.com/27-072015-le-bilan-paradoxal-de-lacampagne-de-noix-de-cajou-2015-enafrique-de-louest

Salifou MI, Masawe PAL. 2018. Guide on Developing Cashew Varieties and Improved Planting Materials. GIZ/ComCashew, Accra, p. 43.

Tandjiékpon MA. 2010. Analyse de la chaine de valeur du secteur anacarde du Bénin. GTZ/iCA, Natitingou, p. 64.

Yidana JA. 2003. Regional distribution and characteristics of some promising cashew mother trees in Ghana. Ghana Journal of Horticulture, 2: 24-30. 